\title{
Erratum to: Factors influencing choice of renal replacement therapy in European Paediatric Nephrology Units
}

\author{
Alan R. Watson • Wesley N. Hayes - Karel Vondrak • Gema Ariceta • Claus Peter Schmitt • \\ Mesiha Ekim • Michel Fischbach • Alberto Edefonti • Rukshana Shroff • Tuula Holta • \\ Aleksandra Zurowska • Gunter Klaus • Sevan Bakkaloglu • Constantinos J. Stefanidis • \\ Johan Van de Walle • European Paediatric Dialysis Working Group \\ Published online: 9 August 2013 \\ (C) IPNA 2013 \\ Erratum to: Pediatr Nephrol \\ DOI 10.1007/s00467-013-2555-z
}

There was an error in the authors name of the article

The correct name is:

Constantinos J Stefanidis

The online version of the original article can be found at http://dx.doi.org/ 10.1007/s00467-013-2555-z.

\footnotetext{
A. R. Watson $(\bowtie) \cdot$ W. N. Hayes

Children's Renal \& Urology Unit, Nottingham Children's Hospital, QMC Campus, Derby Road, Nottingham NG7 2UH, UK

e-mail: judith.hayes@nuh.nhs.uk

K. Vondrak

University Hospital Motol, Prague, Czech Republic

G. Ariceta

Hopital Cruces, Barakaldo, Vizcaya, Spain

C. P. Schmitt

Centre for Paediatric \& Adolescent Medicine, Heidelberg, Germany

M. Ekim

Ankara University Hospital, Ankara, Turkey
}

M. Fischbach

Hospital Hautepierre, Strasbourg, France

\author{
A. Edefonti \\ Pediatrica De Marchi, Fondazione IRCCS Policlinico, Milan, Italy \\ R. Shroff \\ Great Ormond Street Hospital for Children, London, UK \\ A. Zurowska \\ Gdansk University Hospital Medical School, Gdansk, Poland \\ G. Klaus \\ KfH Paediatric Kidney Centre, Marburg, Germany \\ S. Bakkaloglu \\ Gazi University Hospital, Ankara, Turkey \\ C. J. Stefanidis \\ “A \& P Kyriakou”, Children's Hospital, Athens, Greece \\ J. Van de Walle \\ University Hospital, Ghent, Belgium
}

